\title{
Structural Characterization of Supported Metal Nanoparticles
}

\author{
JUDITH C. YANG, FENGTING XU, HUIPING XU, LAURENT MENARD*, KATHRYN GUY*, \\ LIN-LIN WANG**, JOHN SHAPLEY*, ANATOLY FRENKEL***, DUANE JOHNSON**, \\ RALPH NUZZO*
}

Department of Materials Science and Engineering, University of Pittsburgh, 848 Benedum Hall, Pittsburgh, PA 15261.

*Department of Chemistry, University of Illinois at Urbana-Champaign, A128 Chemical \& Life Sciences Lab, 600 South Mathews Avenue, Urbana, IL 61801

**Department of Materials Science and Engineering, 201 Materials Science and Engineering Bldg., 1304 W. Green St., Urbana, IL 61801

*** Department of Physics, Yeshiva University, 245 Lexington Avenue, New York, NY 10016

Very small metal clusters can exhibit patterns of reactivity and catalytic activity that are dramatically distinct, and sometimes completely opposite, than behaviors seen with larger clusters. It therefore remains a significant need in research to fundamentally understand and predict the local structure and stability of catalytic materials. Surprisingly, supported nanoclusters that are used in heterogeneous catalysis are described frequently in qualitative terms (e.g. oblate, or raft-like, or truncated polyhedral). These types of descriptions miss the essential details of the atomic scale structures, the dynamics they embed, and the energy landscapes that define their transformation. Two complementary state of the art methodologies, quantitative scanning transmission electron microscopy (STEM) and synchrotron X-ray absorption fine-structure (XAFS) methodologies will be utilized and specially designed for determining the three-dimensional (3D) structure and structural habits, both individually and as an ensemble, critical for understanding metallic nanoclusters. The various nanoparticles were characterized with HAADF imaging as well as by energy dispersive Xray spectroscopy (EDS) on a dedicated STEM, and high-resolution electron microscopy (HREM). The experimental work will be integrated with theoretical calculations to help interpret and accelerate identification of cluster bonding motifs, possible metastable structures, and determining electronic properties and reactivity of relevant clusters.

Systems of interest include both model materials prepared in the form of ligand-stabilized clusters as well as supported materials of technological interest. We choose $\mathrm{Au}, \mathrm{Pt}$ and $\mathrm{PtRu}_{5}$ nanoclusters as model systems, as well as $\mathrm{Pd}-\mathrm{Cu}$ since it has recently emerged as an exciting approach to remove nitrates from drinking water because of their high activity for nitrate reduction. Small, monodisperse $\mathrm{Au}_{13}$ clusters have been prepared via a ligand exchange reaction of alkanethiols onto phosphinehalide gold clusters. In one synthetic protocol, two fractions separated with column chromatography exhibit very different UV-visible spectroscopy. Transmission electron microscopy and EXAFS revealed that the cluster exhibiting a molecule-like UV-visible spectrum was found to have a local structure consistent with an icosahedral $\mathrm{Au}_{13}$ core while the fraction with a smoothly varying UVvisible absorption spectrum was determined to consist of larger cuboctahedral Au clusters (average core consisting of 180 atoms). Furthermore, the effects of post-treatments to remove the ligands were investigated on $\mathrm{Au}_{13}$ nanoparticles on $\mathrm{TiO}_{2}$ support. Both the ozone post-treatment and thermal annealing lead to sintering of the $\mathrm{Au}_{13}$ nanoparticles, but the ozone yielded less $\mathrm{Au}$ nanoparticles 
sintering than conventional thermal treatment. The truncated cuboctahedral structure of $\mathrm{Au}$ nanoparticles is observed in ozone treatment process while the thermal treatment results in mostly cuboctahedral structure. $\mathrm{PtRu}_{5}$ bimetallic clusters were also investigated by combined HREM, STEM and XAFS along with modeling. Combined HREM, STEM and XAFS revealed that the $\mathrm{PtRu}_{5}$ structure on $\mathrm{C}$ black was a truncated cuboctahedron, with $1.5 \mathrm{~nm}$ diameter, and Pt segregation to the surface. Modeling revealed that the Pt segregation is due to an electronic size effect, and the bond disorder, noted by XAFS, is due to the support interaction with a nanoparticle.

We also studied $\mathrm{Pd}-\mathrm{Cu}$ bimetallic catalysts because of its promising chemistry for nitrate reduction for purification of drinking water. We have systematically synthesized $\mathrm{Pd}-\mathrm{Cu}$ nanoparticles (with $\mathrm{Pd}-\mathrm{Cu}$ ratios of 50:50 to 90:10) using colloidal chemistry methods. Interestingly, the average particle size for specific composition abruptly changes from near $5 \mathrm{~nm}$ for the 80:20 (Pd:Cu) colloid to near $3 \mathrm{~nm}$ for the 70:30 colloid due to a change in structure (FIG.1a). All of the Pd-Cu ratios showed a narrow size distributions in the range of 3 5nm (FIG. 1b). HREM images reveal that the particles with $70 \%$ or lower amounts of Pd form a core-shell structure, whereas for the 80 and $90 \% \mathrm{Pd}$ particles, these are a face-centered cubic (FCC) structure containing a homogeneous mixture of $\mathrm{Pd}$ and $\mathrm{Cu}$. Intriguing initial studies of the chemical properties of the $\mathrm{Pd}-\mathrm{Cu}$ nanoparticles indicate some differences in the chemical activity for nitrate reduction due to synthesis method. We have noted that the nitrate reduction capabilities of the alloy materials depends as on the Pd:Cu ratio as 50:50> $60: 40>>70: 30,80: 20,90: 10$ where no reduction is seen in the last three cases, at least at room temperature

This research program is supported through the DOE-BES Catalysis Program (DEFG0203ER15476). The Materials Research Laboratory at Univ. of Illinois at Urbana-Champaign (UIUC) was used for the electron microscopy, which is a DOE (DEFG02-96-ER45439) supported facility. The EXAFS experiments were performed at Brookhaven National Laboratory. The University of Pittsburgh's Mascaro Sustainability Initiative supports the electron microscopy effort on the development of catalysts for purification of drinking water. The water purification research effort at UIUC is funded through a NSF-supported water center (CAMPWS) at Illinois.
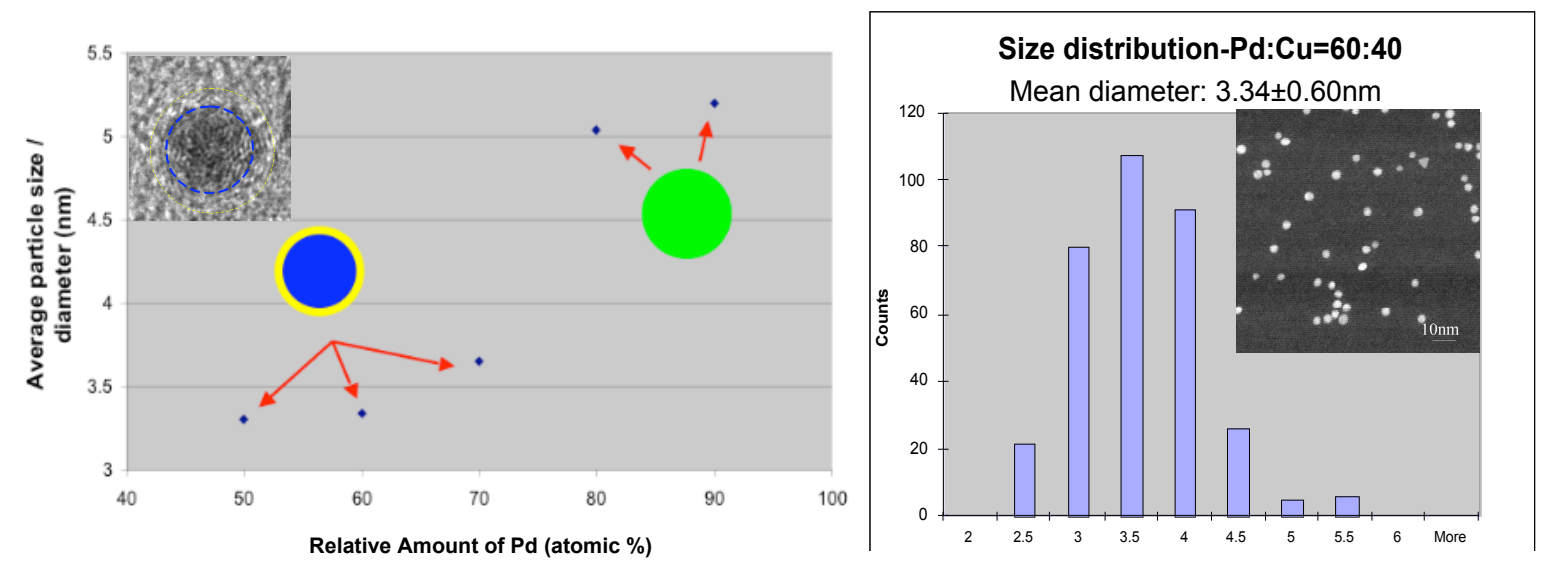

FIG. 1. Pd;Cu bimetallic catalysts where (a) the average particle diameter abruptly changes between $\mathrm{Pd}: \mathrm{Cu}$ ratios of $70: 30$ and 80:20, corresponding to a change of core-shell structure (inset) to homogeneous alloy and (b) a narrow size distribution is always noted, such as in the 60:40 Pd:Cu nanoparticles. 